\title{
Challenging low Reynolds - SWT blade aerodynamics
}

\author{
Damian Kądrowski ${ }^{1, *}$, Michat Kulak ${ }^{1}$, Michał Lipian ${ }^{1}$, Małgorzata Stępień ${ }^{1}$, Piotr Baszczyński ${ }^{1}$, Karol Zawadzki ${ }^{1}$ and \\ Maciej Karczewski ${ }^{1}$ \\ ${ }^{1}$ Institute of Turbomachinery, Lodz University of Technology, 90 - 924 Lodz, 219/223 Wolczanska Street, Poland
}

\begin{abstract}
One of the main issues related to the design and development of small wind turbines (SWTs) is the low Reynolds number. Operation in the transitory regime makes the rotor aerodynamic analysis a challenging task. Project GUST (Generative Urban Small Turbine) realized currently at the Institute of Turbomachinery (Lodz University of Technology, Poland) is devoted to the development of SWT $(D=1.6 \mathrm{~m})$ for low-Reynolds number (low wind speed) flow conditions. The emphasis is on the blade design, aiming at improving the rotor aerodynamic efficiency. The paper will highlight the rotor design process, based on contemporary methods of experiment-simulation integration approach and use of rapid manufacturing techniques. In-house wind tunnel measurements of a scaled model performance were executed. A numerical analysis using dedicated software (QBlade) was conducted in parallel. A comparison between the obtained results indicated that the chosen numerical tools are capable of providing a reliable output, even in complex, transitional flow conditions. Bearing in mind the above observations, QBlade was incorporated into the development process of a completely new blade geometry which would increase rotor performance. The selected design has indeed prove to show better power outcome in an additional experimental campaign.
\end{abstract}

\section{Introduction}

Nowadays, the objective of fulfilling the world energy requirements and at the same time of taking into account global climate changes is a challenging task. The aim is for traditional, non-renewable power sources to be aided and ultimately replaced by sustainable ones. Although wind energy, as an omnipresent and renewable source, is a promising solution, its utilization in large scale wind turbines, frequently organized into big wind farms, can negatively affect both climate and atmosphere [1]. Herein, the dispersed and decentralized small scale wind turbines show a significant potential for power production. It can be sufficient for urban applications or domestic needs without influencing the weather and environment conditions [2].

It is essential to understand small wind turbine characteristics, while having in mind their drawbacks. One should take into consideration such difficulties as: relatively high initial price, the need for proper site assessment resulting in optimal wind turbine placement [3], recognition of wind conditions on site (i.e. wind velocity, wind direction, temporal conditions) [4], national policy and aero-acoustic noise. On the contrary, when placed and sized correctly and operating at design conditions, small wind turbines can be considered as a convenient, effective and environment-friendly power supply. Taking economics into account, two factors are of concern: initial cost per $\mathrm{kW}$ power and the unit cost per $\mathrm{kWh}$ it produces [2].
To address the above issues, students' project GUST (Generative Urban Small wind Turbine), focuses on building horizontal axis wind turbine of $2 \mathrm{~m}^{2}$ swept area and nominal power around $750 \mathrm{~W}$. The team has presented the prototype during the International Small Wind Turbine Contest, being its winner for three times in a row (2016-2018). Besides the self-constructed mechanical components (such as hydraulic brake), alterations of power generator (system of coils switching), as well as self-designed safety and control systems, the most important innovation proposed by GUST is related to the aerodynamic design of wind turbine blades followed by their production through rapid prototyping (3D printing) methods.

The aim of the paper is to present a rotor design process. It is based on experiment-simulation integration (utilizing the in-house wind tunnel and simulation in dedicated software - Qblade). A correspondence between the obtained results indicates that the selected numerical tools are capable of granting a reliable output, even though the flow is complex (conditions of laminarturbulent transitional flow). As a next step, QBlade was incorporated into the process of developing completely new wind turbine blade geometry, aimed at increasing rotor performance. The selected design was subjected to an additional experimental process, in order to prove the better power outcome.

\footnotetext{
*orresponding author: damian.kadrowski@gmail.com
} 


\subsection{Reynolds number definition - flow similarity criterion}

In order to consider the air movement around the aerofoil it was necessary to estimate the Reynolds numbers in particular cross-sections (25 uniformly distributed stations along blade span):

$$
\operatorname{Re}=u c_{i} / v_{\text {air }},
$$

where $c_{i}$ denotes assumed chord length of a specific cross section, $v_{\text {air }}$ is kinematic viscosity of the air and $u$ represents a vector sum of axial wind speed and rotational speed of the blade element on the analysed radial position. Note that, in order to simplify the analysis at this early stage, the induced velocity is not taken into account. The estimation resulted in Reynolds number values in the range of $50000 \div 150000$.

It is also possible to define the global Reynolds number basing on the rotor overall size, whence $\mathrm{c}$ in numerator becomes $D$, the rotor diameter. This criterion is especially useful when comparing rotors of different sizes or subjected to different wind velocity.

In the current study the default Reynolds number is the local one, based on chord length $c$, unless explicitly specified.

\section{Numerical analysis - Qblade}

QBlade is open source wind turbine calculation software, integrating various codes and models for a thorough simulation of wind turbine performance [5]. It integrates tools for e.g. aerofoil design and evaluation, BEM rotor simulation, simplified structural analysis of the blade and results post-processing.

The performed aerodynamic study included complex blade and rotor geometry design and analysis. The inhouse designed blade, originally inspired by the model from NTNU Norway [6] is based on NREL S826 and S834 aerofoils. As a result of the differences in the rotor diameters between the two rotors, the original chords were scaled by a factor defined as a ratio between modified and original rotor radius.

The main criterion considered during the design process was to maximize the Annual Energy Production (AEP). Having the wind conditions imposed [6], the whole construction needed to be adjusted to the specific set of parameters. The geometry was also optimised to lower the cut-in wind speed and widen the operational range of the Tip Speed Ratios (TSRs, formula (4)). It is important to point out that the rotor geometry is characterized by relatively small scales (notably chord length). Thus the blade design criteria should also take into account the mechanical limitations of materials load concentration areas.

An initial part of the procedure was the aerofoil selection. The NREL S820 and S830 aerofoil families are dedicated for small wind turbines [7], having in mind the low Reynolds number observed for the flow. The assessment of the profiles' performance was done with the QBlade-integrated XFoil software, a panel-methodbased code for inviscid and viscous flow analysis around aerofoils [8]. Further analysis and the validation process depended on data acquired from XFoil polars, visible in Fig. 1 (S826) and Fig. 2 (S834). The plots show $\mathrm{Cl} / \mathrm{Cd}$ (lift-to-drag ratio) as a function of angle of attack $(A o A)$ and $C l$ versus $C d$. The simulations were performed for different Reynolds numbers, corresponding to local values observed at different blade stations. The flow is considered incompressible (i.e. $M a \approx 0$ ).

In every figure all considered curves share roughly the same trace. However, it is noticeable how important the influence of the applied $R e$ is. With decreasing Reynolds number the aerofoil performance falls drastically. In case of S826 aerofoil, for $R e=62 \mathrm{k}$ maximum $C l / C d \approx 41$, while for $R e=31 \mathrm{k}$ maximum $C l / C d \approx 20$; this means that by doubling the Reynolds number it is possible to double the aerofoil performance. A closer analysis reveals that for both aerofoils the maximum $C l / C d(A o A)$ is roughly proportional to $R e$.
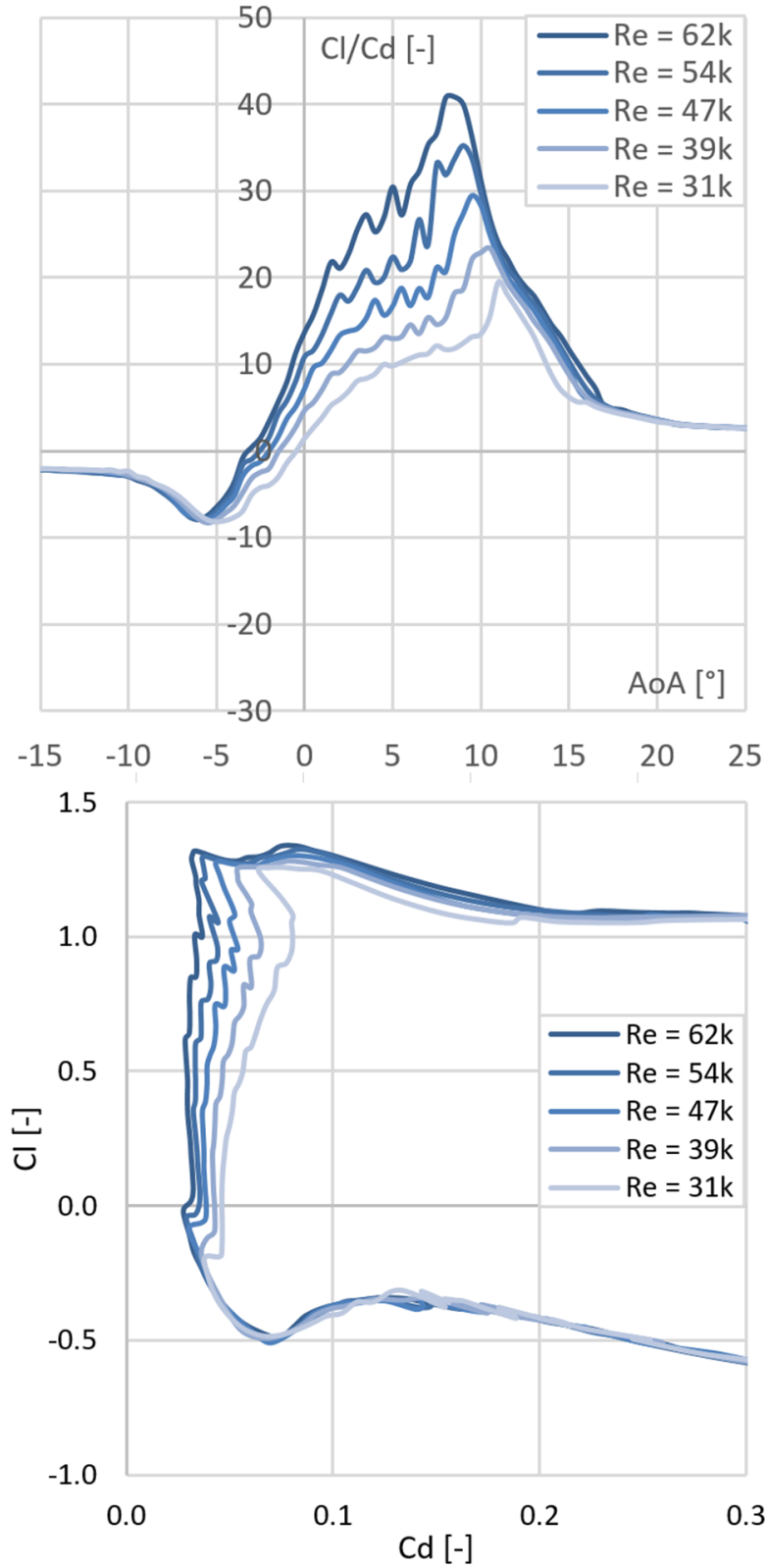

Fig. 1. S826 aerofoil polar plots: $C l / C d(A o A)$ (top) and $C l(C d)$ (bottom). 


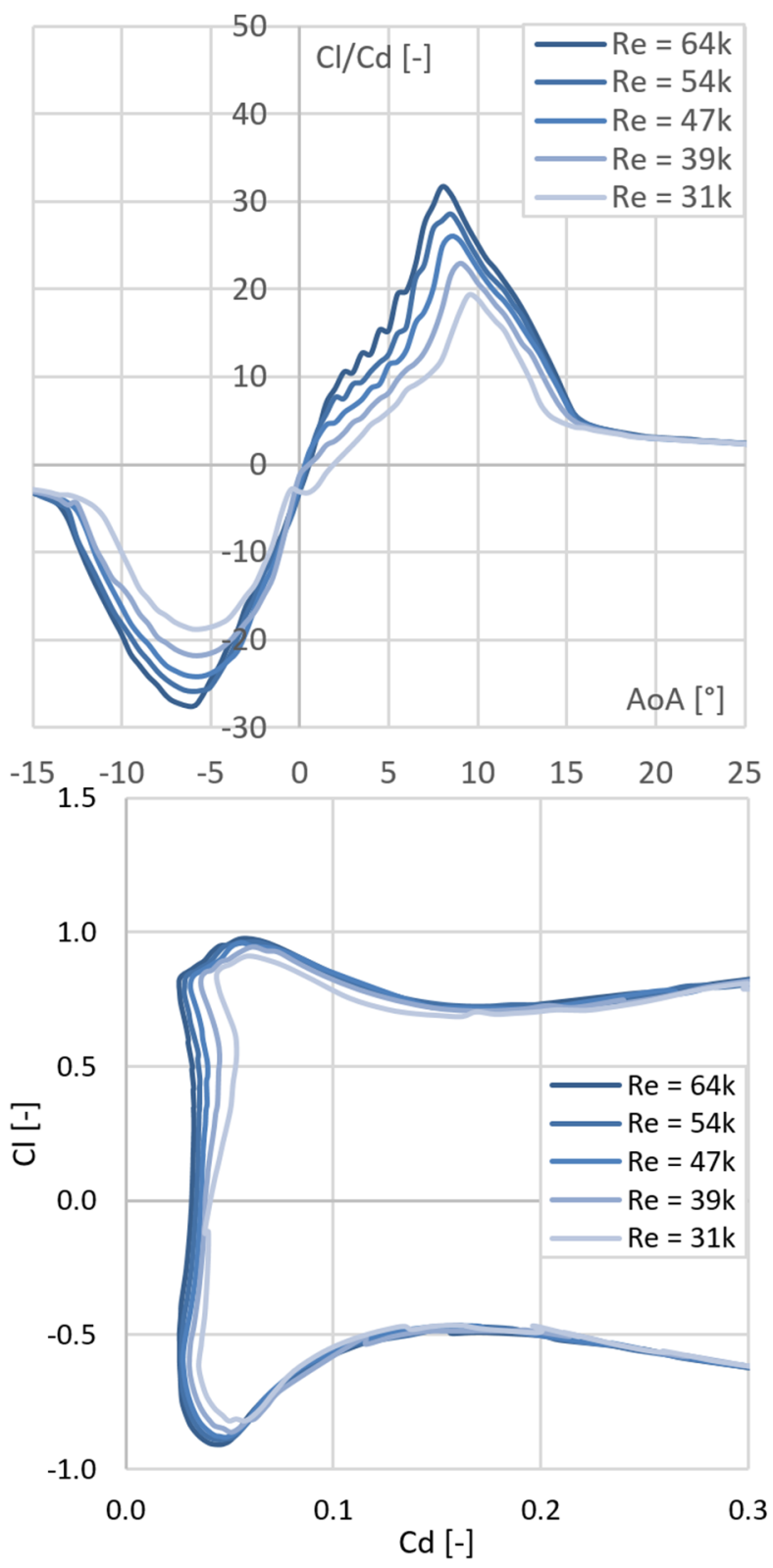

Fig. 2. S834 aerofoil polar plots: $C l / C d(A o A)$ (top) and $C l(C d)$ (bottom).

The same may be stated for the $A o A$ corresponding to maximum lift to drag ratio, which value is shifted towards higher values as Reynolds number decreases.

It is also visible that the actual numerical analysis is difficult to conduct properly, judging by the unstable traces of the presented characteristics. This issue may be addressed by interpolating and smoothing the obtained results, however it is important to know of its presence.

Taking into account the rotor aerodynamic performance, it would be best to use S826 along the entire blade span (as it displays the highest lift-to-drag ratio). Thus this aerofoil was applied in the middle and tip sections of the blade. However, due to its relatively low thickness, S826 may be unfit for blade regions exposed to high loads, such as root. To ensure sufficient stiffness of the blade in that region, a higher $b / c$ ratio aerofoil was chosen, that is S834. Selected aerofoil properties are given in Table 1, while the two selected profiles can be seen in Fig. 3.
The blade was constructed on the basis of the existing geometry mentioned above, modified using numerical tools: CFD solver (ANSYS package) and simplified codes (FAST, XFoil). A parallel examination in Qblade included examination of rotor performance over a range of wind speeds and TSR (2-7). The obtained blade design was validated in an experimental process, as presented in the following section.

Table 1. Features of selected NREL low-Re aerofoils.

\begin{tabular}{|l|c|c|c|c|}
\hline & $\mathbf{S 8 2 2}$ & $\mathbf{S 8 2 3}$ & $\mathbf{S 8 2 6}$ & $\mathbf{S 8 3 4}$ \\
\hline Thickness-to-chord ratio $\boldsymbol{b} / \boldsymbol{c}$ & 0.16 & 0.212 & 0.14 & 0.15 \\
\hline Max $\boldsymbol{C} / \boldsymbol{C d}$ & 42.84 & 42.52 & 60.72 & 47.42 \\
\hline Corresponding $\boldsymbol{A o} \boldsymbol{A}\left[^{\circ}\right]$ & 8.4 & 9.1 & 8.3 & 7.1 \\
\hline Corresponding $\boldsymbol{C}$ & 0.921 & 1.157 & 1.409 & 0.909 \\
\hline Corresponding $\boldsymbol{C d}$ & 0.022 & 0.027 & 0.023 & 0.019 \\
\hline
\end{tabular}

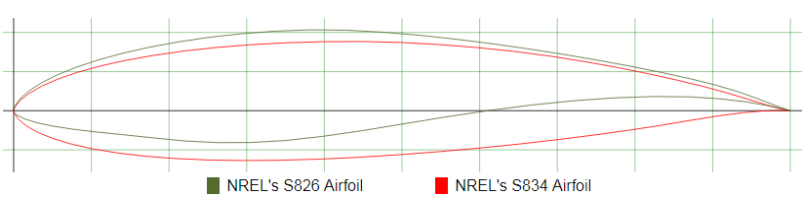

Fig. 3. NREL S826 (green) and S834 (red) aerofoils.

\section{Wind tunnel test}

The experimental examination of SWT scaled model was conducted at the subsonic, open-test section wind tunnel of the Institute of Turbomachinery, Lodz University of Technology (IMP TUL). The reference velocity value was provided by pneumatic measurements. Prandtl-type Pitot tube was used to measure dynamic pressure at the outlet of the tunnel. The ambient temperature, atmospheric pressure and humidity were used to compute the air density. The whole experimental method aims to evaluate the wind turbine performance $c_{p}$ as a function of the tip-speed ratio (rotor blade tip velocity divided by wind freestream velocity). Wind turbine speed was actively regulated by a motorgenerator connected to a microcontroller in order to control precisely the device rotational velocity. The latter, along with the shaft torque (measured with a torquemeter) provided information concerning the rotor aerodynamic performance. The stages of the experimental procedure are presented in Fig. 4.

The first stage was to complete and prepare the test bench, before commencing the research. Afterwards, the behaviour of the free stream was investigated at selected distances from the outlet, to determine the optimum location of the turbine model in the aerodynamic tunnel. In the next steps, the measurement technique and approach were verified, and then followed the actual study of selected wind turbine models. The results were further recomputed and interpreted, which included determining the uncertainty, the characteristics and conclusions made from the research. A view of the measurement site after mounting the turbine and connecting the measuring apparatus is shown in Fig. 5. Referring to the annotations, the measurement setup 
consisted of: 1 - rotational speed meter, 2 - digitalanalogue converter and data acquisition module, 3 differential pressure sensor, 4 - temperature-relative humidity sensor, 5 - reference pressure sensor, 6 - rotor, 7 - torque meter, 8 - motor, 9 - servo motor controller, 10 - Prandtl pressure probe.

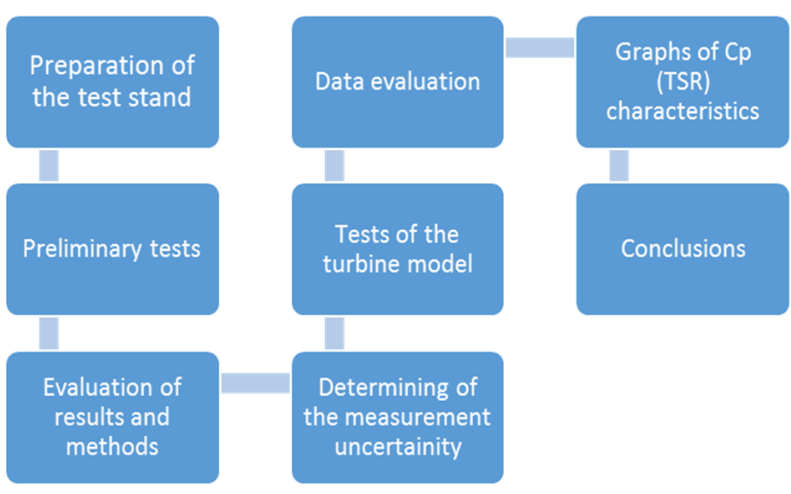

Fig. 4. Sample procedure of wind turbine characteristic experimental assessment.

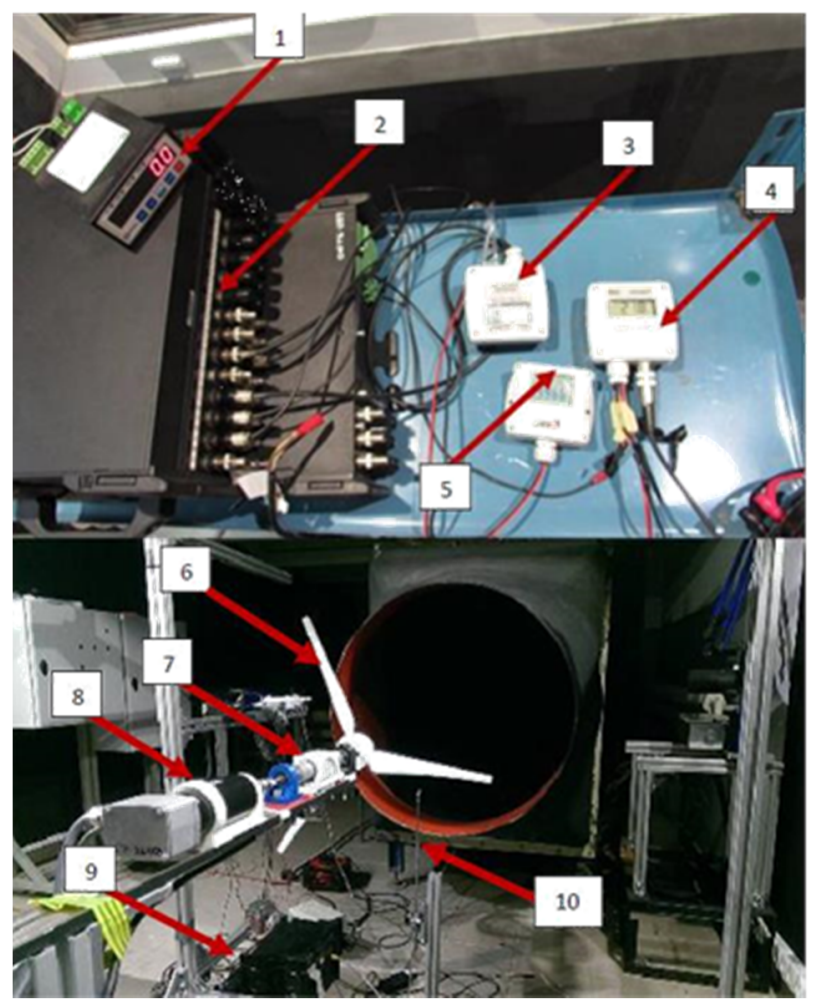

Fig. 5. Wind turbine test bench in IMP TUL wind tunnel.

The measurement procedure commences with setting the flow velocity $v$ and turbine rotational speed $\omega$ at the desired values. Data is collected from all transducers (temperature $T$, humidity $R H$, dynamic pressure $p_{d}$, atmospheric pressure $p_{a}$, turbine shaft torque $M$, rotational speed $\omega$ ). Flow velocity is computed as:

$$
v=\sqrt{2 p_{d} / \rho}
$$

with flow density $\rho$ corrected for the thermodynamic properties of flow. Rotor power is computed as:

$$
P=M \omega \text {. }
$$

Dimensionless parameters - tip speed ratio $T S R$ and power coefficient $c_{p}$ - are computed as:

$$
\begin{gathered}
T S R=\omega R / v, \\
c_{p}=P /\left(p_{d} v \pi R^{2}\right),
\end{gathered}
$$

where $R$ is the rotor diameter.

The procedure was repeated over a desired range of $v$ and $\omega$ to trace the rotor characteristic. The tests of the scaled wind turbine model were performed in order to verify the results obtained in the numerical simulation process. The models used in the measurements had a diameter of $450 \mathrm{~mm}$ and $400 \mathrm{~mm}$. They were manufactured through $3 \mathrm{D}$ printing, an emerging method offering excellent repeatability at relatively low cost and time expenses. The datasets were determined separately for each wind speed; the data was approximated by a $4^{\text {th }}$ degree polynomial to accurately reflect the outline of the curve.

At a specific wind speed, the power obtained by the rotor is mainly dependent on the relative speed between the end of the blade and the wind speed (the tip-speed ratio). Both too low and too high TSR results in adverse interaction between rotor and flow. Consequently, part of the wind kinetic energy remains unexploited and/or the rotor performance decreases. Thus each rotor has an optimal tip-speed ratio for which the wind turbine performance attains its maximal value.

\section{Results and discussion}

These aforementioned characteristics can be seen in Fig. 6. This graph shows the wind turbine performance $c_{p}$ as a function of wind turbine rotational velocity and wind speed (TSR) for different wind velocities (different Reynolds number calculated based on the rotor diameter). The tested rotor has a diameter of $450 \mathrm{~mm}$. Both experimental and numerical results are presented.

As it was expected, higher $R e$ allows the turbine to reach higher $c_{p}$ values. One can see that both experimental and numerical results are coincident for sub-optimal rotational velocities $(T S R<2.5)$. The differences appearing for TSR between 2.5 and 4 may be caused by uncertainty in calculating TSR in the experiment. Along the whole range of rotational velocities, there is a good correlation between simulation and experiment for $R e=360 \mathrm{k}$ and $R e=420 \mathrm{k}$. The discrepancies noticeable for $R e=300 \mathrm{k}$ may result from the fact, that it corresponds to the aerofoil transitional region, where its performance is especially difficult to assess or predict.

One should bear in mind that the local Re choice for aerofoil characteristics is based on arithmetic average of its predicted values along the entire blade span for optimal TSR; therefore, the discrepancies between the values of optimal TSR may result from the necessity of local $R e$ estimation as a fixed value for the whole blade span. This approach was selected by the authors on the basis of previous experience. 


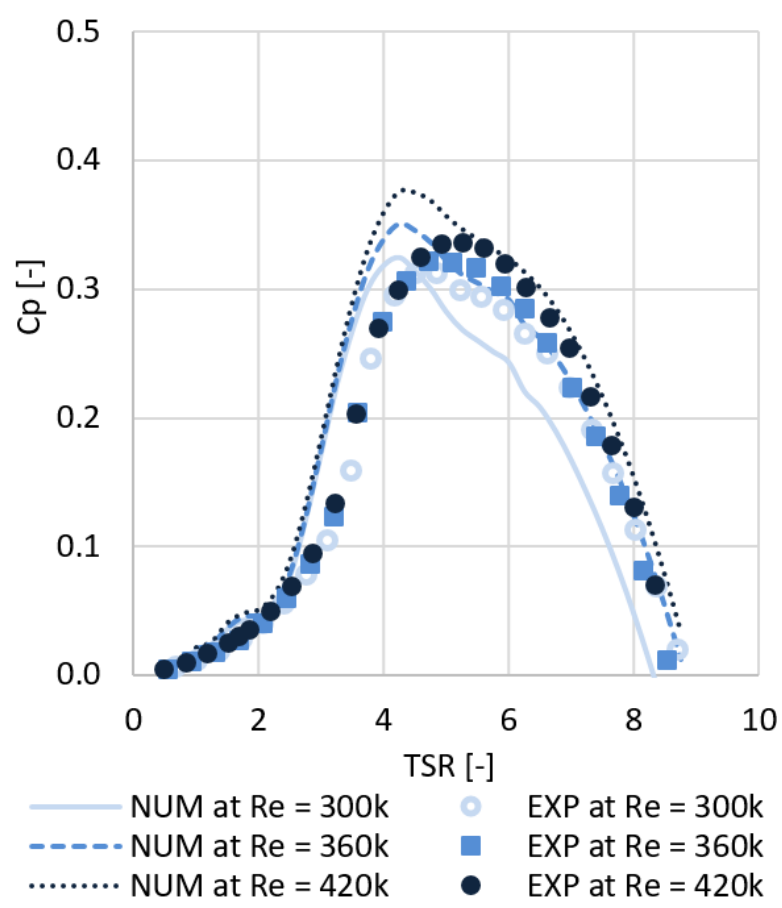

Fig. 6. $C_{p}(T S R)$ characteristics for different $R e$; simulation vs. experiment for NREL-based blade.

The value of rotor idle speed (at which $c_{p}=0$ ) is in agreement between the experimental and numerical results (especially for higher $R e-360 \mathrm{k}$ and 420k).

Quantitatively, the differences between simulated and experimental optimal TSR are in the range of $10 \%$ to $15 \%$, maximum $c_{p}$ differences lie in range of $5 \%$ to $10 \%$.

It can be concluded that the proper choice of Reynolds number for profile characteristics determination is the most crucial aspect; if chosen too low it may result in significant underprediction of wind turbine performance; if chosen too high - the performance may be overpredicted. Nonetheless, the curve shape could be preserved.

Summing up the above, it may be stated that three main areas of interest have been addressed:

- QBlade applicability in wind turbine (existing) blade analysis at low $R e$ was confirmed;

- Verification of the correctness of numerical model assumptions and simplifications was performed; the model limitations were determined;

- Quantification of the level of correspondence of the results in relation to the experiment was carried out and proved to give satisfactory results as far as the design process is concerned.

On the basis of the above observations, we undertook to establish a more aerodynamically efficient blade. In order to do that a new set of aerofoils was chosen, the Wortmann FX series for low-Reynolds applications [9]. The chosen aerofoil, FX 63-137, has a significantly higher camber, as compared to S826. The newly designed rotor was tested in the IMP TUL wind tunnel and compared with the original design (Fig. 7). However, this time the models have a diameter of 400 $\mathrm{mm}$.

It can be seen that the experimental optimal TSR is very well predicted by the simulation, as is the maximum $c_{p}$ value. However, there are significant dissimilarities in $T S R$ region above optimal. We should note that all geometrical features were scaled down with respect to earlier considerations; while the printing resolution remained the same. Thus, the quality of model representation deteriorated, especially in regions where small geometrical features are present (such as the blade tip). Also, due to scaling down the chord length, rigidity of the blade, influenced by the thickness, decreased. At increased tip speed ratio (above optimal value) it resulted in sudden drop of performance, due to increasing mechanical efforts. Note that the slenderness and susceptibility to deformation of the newly designed blade was relatively lower than in the previous model, thus the EXP-NUM discrepancies are less significant in this case.

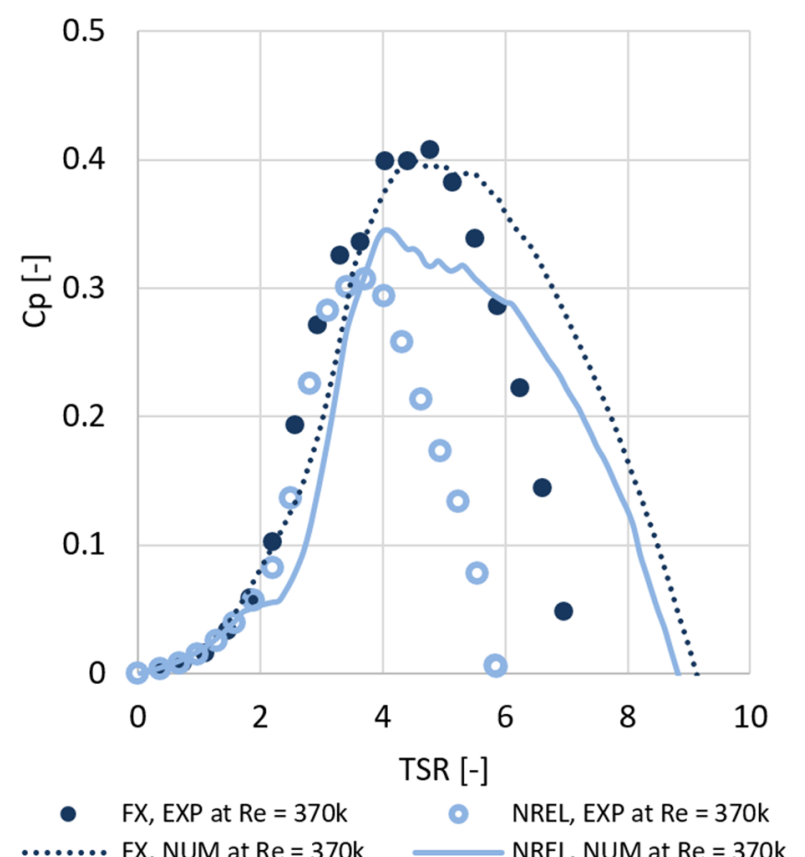

Fig. 7. $C_{p}(T S R)$ characteristics for the same $R e$; simulation vs. experiment for different blade geometries.

Comparing the experimental data for the NRELbased blade at $R e=360 \mathrm{k}, 370 \mathrm{k}$ it is visible that the optimal TSR differs between the two cases, even though $R e$ stays roughly the same. This was attributed to the possible issues with geometrical similarity preservation. Once again, the $3 \mathrm{D}$ printing process resolution is the same in both cases. Thus, the obtained relative blade surface roughness is higher for the smaller rotor; also, the quality of blade geometry is lower, as noted above.

It is interesting to point out the significant gain in the performance of the newly-designed blade. In the previous considerations QBlade was used in the analysis of a design developed using different techniques. In this case it remained the principal tool for design and investigation. Thanks to that the rotor performance was increased by as much as $15 \%$, and blade stiffness was strengthened. Although it is doubtful that for a full-scale wind turbine that much increase would be observed, the case proves the principle of applicability of using QBlade as both an analysis and design tool. 


\section{Conclusions}

This paper discusses observations concerning the design and assessment of wind turbine rotors in low-Reynolds number conditions.

The presented data firstly concerns basic blade geometry analysed experimentally (IMP TUL wind tunnel) and numerically (QBlade). It showed the importance of Reynolds number value for SWTs. It also proved that QBlade is a useful tool for assessment of existing rotor blade results. In order to perform a more thorough analysis it is advisable to supplement it with an experimental, independent study method.

In the second part of the paper a new blade design was discussed, based on a new set of aerofoils and designed entirely using the QBlade software. The results seem very promising, as the model tested experimentally showed $c_{p}$ increase by as much as $15 \%$. This proves the interest in incorporating the simplified model in the design process.

An important conclusion also comes from comparing two rotors of (supposedly) the same geometry, but manufactured in different scales. One should have in mind that especially in the case of small wind turbines, low Reynolds is connected with low-size geometry scales; therefore the Reynolds number similarity criterion may be insufficient. The described technology of manufacturing (3D printing) offers the perfect repeatability of produced objects (blades). However, the scaling of the models may lead to deterioration in the quality of small features representation, what in turn leads to unanticipated geometrical similarity discrepancies. This will project on discrepancies between simulation and the actual, empirical results.

\section{References}

1. C. Wang, R. Prinn, Potential climatic impacts and reliability of very large-scale wind farms, Atmos. Chem. Phys., 10, 2053-2061 (2010)

2. A. Tummala, R. Velamati, D. Sinha, V. Indraja, V. Krishna, A review on small scale wind turbines, Renew. Sustain. Energy Rev., 56, 1351-1371 (2016)

3. B. Grieser, Y. Sunak, R. Madlener, Economics of small wind turbines in urban settings: an empirical investigation for Germany. Renew. Energy, 78, 334350, (2015)

4. K. Sunderland, T. Woolmington, J. Blackledge, M. Conlon, Small wind turbines in turbulent (urban) environments: A consideration of normal and weibull distributions for power prediction, J. Wind Eng. Ind. Aerodyn., 121, 70-81, (2013)

5. http://www.q-blade.org/ (20.07.2018)

6. L. Sætran, J. Bartl, Invitation to the 2015 "Blind test 4" Workshop, Technical document NTNU, www.sintef.no/globalassets/project/nowitech/events/ bt4_invitation2015.pdf (15.07.2018)

7. https://wind.nrel.gov/airfoils/AirfoilFamilies.html (18.07.2018)
8. http://web.mit.edu/drela/Public/web/xfoil/ (18.07.2018)

9. H. Riblett, What Are Wortman Airfoils??, J. Sport Aviation, Nov. 1988 